\title{
Auswirkungen von Enhanced Annuities auf den Bestand eines Versicherers
}

\author{
Von Gudrun Hoermann, St. Gallen \\ und Jochen RuB, Ulm ${ }^{1}$
}

Inhaltsübersicht

1. Einführung

2. Modellierung

2.1 Die Bestimmung der Leistungsdauer

2.2 Die Bestimmung der Leistungshöhe

2.3 Gewinn pro Police und im Portfolio

3. Auswertungen

3.1 Wahl der Parameter und Simulation

3.2 Ergebnisse

4. Zusammenfassung

\section{Einführung}

Rentenversicherungen, bei denen die Rentenhöhe nur vom zu verrentenden Betrag, vom Geschlecht und vom Alter des Versicherten bei Rentenbeginn abhängt, diskriminieren typischerweise Personen mit verminderter Lebenserwartung, da diese für den selben Beitrag im Durchschnitt geringere Leistungen erhalten.

Zur Veranschaulichung dieser Aussage vergleichen wir mögliche Leistungen einer Risikolebensversicherung mit den Leistungen einer Rentenversicherung (bei gegebenem Einmalbeitrag), und zwar in Abhängigkeit des Gesundheitszustands des jeweiligen Versicherungsanwärters. Eine Person, deren Gesundheitszustand zum Zeitpunkt der Gesundheitsprüfung schlechter als der Durchschnitt erachtet wird, stellt ein höheres Risiko für den Lebensversicherer dar. Daher erhält diese Person bei gleicher Prämie eine geringere Leistung als ein Versicherter mit durchschnittlicher Lebenserwartung bei Vertragsabschluss. Einen so genannten „preferred“ Tarif, d. h. eine erhöhte Leistung bei gleicher Prämie, könnte ein Lebensversiche-

1 Gudrun Hoermann, Universität St. Gallen, Institut für Versicherungswirtschaft. Email: gudrun.hoermann@unisg.ch. Jochen Ruß, Institut für Finanz- und Aktuarwissenschaften. Email: j.russ@ifa-ulm.de. 
rer einem Versicherungsanwärter bieten, der gesünder ist als der Durchschnitt. Produkte, bei denen das Verhältnis von Prämie zu Leistung für „gute Risiken“ geringer ist, sind mittlerweile in vielen Versicherungsmärkten für Risikoleben- und Berufsunfähigkeitsversicherungen üblich. Rentenversicherer bieten hingegen in der Regel in allen drei Fällen eine so genannte Standardrente, welche lediglich von Alter, Geschlecht und Einmalbeitrag abhängt. Versicherte Personen mit gutem Gesundheitszustand, d. h. hoher Lebenserwartung, werden somit bevorteilt, da sie die Standardrente voraussichtlich über einen längeren Zeitraum als Versicherte mit durchschnittlicher Lebenserwartung beziehen können. Für Personen mit unterdurchschnittlicher Lebenserwartung ist die Rente jedoch $\mathrm{zu}$ gering und damit in diesem Sinne unfair kalkuliert.

Das Preis-Leistungs-Verhältnis herkömmlicher Rentenversicherungen ist also umso besser, je höher die Lebenserwartung des Versicherten. Dies könnte u. a. eine Ursache für das so genannte „annuity puzzle“ darstellen. Hierunter versteht man das Paradoxon, dass lediglich ein geringer Anteil der Senioren eine Rentenversicherung besitzt ${ }^{2}$, obwohl unter gewissen Annahmen gezeigt werden kann, dass eine (teilweise) Verrentung ökonomisch vorteilhaft ist (vgl. Brown et al. (2005)). Brown et al. (2005) gehen jedoch implizit davon aus, dass das Preis-Leistungs-Verhältnis von Renten für alle potentiellen Versicherten im Wesentlichen gleich ist, da sie annehmen, dass zukünftige Sterbewahrscheinlichkeiten lediglich von Alter und Geschlecht der Person abhängen. Man kann jedoch beobachten, dass die Lebenserwartung von Rentenversicherten deutlich über der durchschnittlichen Lebenserwartung der Gesamtpopulation liegt. Personen, die ein faires Preis-Leistungs-Verhältnis erhalten, verrenten somit häufiger als Personen mit geringer Lebenserwartung.

Durch steuerliche Aspekte wird diese Problematik weiter verstärkt. Das im Januar 2005 in Deutschland eingeführte Alterseinkünftegesetz incentiviert den Bezug von lebenslangen Rentenleistungen aus einem Altersversorgungsvertrag gegenüber einer Einmalzahlung. Während Versicherte mit reduzierter Lebenserwartung in der Vergangenheit ohne steuerliche Nachteile eine Kapitalabfindung wählen konnten, stehen sie nun vor der Wahl zwischen einer zum Teil steuerschädlichen Einmalzahlung oder einer Verrentung zu unfairen Konditionen. Darüber hinaus existieren teilweise staatlich geförderte oder steuerbegünstigte Produktlinien, bei denen eine Verrentung Pflicht ist. In diesem Fall sind Personen mit erhöhter Sterblichkeit sogar gezwungen, zu nachteiligen Bedingungen zu verrenten, $d$. h. der Barwert der Rentenleistungen kann signifikant geringer als der zu verrentende Betrag sein.

2 Gemäß Dushi, I. und Webb, A. (2004) haben beispielsweise nur $10.2 \%$ der Senioren in den USA einen Teil ihres Vermögens in Rentenversicherungen investiert. 
Aus ökonomischer Sicht ist es grundsätzlich sinnvoll, Anreize zur Verrentung zu schaffen; wenn jedoch am Markt lediglich traditionelle Rentenversicherungsprodukte angeboten werden, ergeben sich durch solche Regelungen erhebliche Nachteile für Versicherte, die zu Rentenbeginn eine unterdurchschnittliche Lebenserwartung besitzen.

Die beschriebene Nichtberücksichtigung des Gesundheitszustands als Tarifierungsmerkmal und die daraus resultierende Benachteiligung „kranker" Versicherter könnte durch das Angebot so genannter Enhanced Annuities $^{3}$ beseitigt werden. Darunter versteht man Rentenversicherungen, bei denen die Rente umso höher ist, je geringer die Lebenserwartung der versicherten Person. Enhanced Annuities bieten somit idealer Weise ein gleiches Preis-Leistungs-Verhältnis für alle Kunden. Damit sollte auch unter denjenigen, die derzeit eine Verrentung ablehnen, die Akzeptanz von Renten steigen. Auf dem deutschen Versicherungsmarkt gibt es bisher nur sehr wenige derartige Produkte. ${ }^{4}$

In der praktischen Umsetzung wird bei Rentenbeginn eine Gesundheitsprüfung bzw. ein individuelles Underwriting durchgeführt. Daraus ergeben sich die zur Kalkulation der Rentenhöhe verwendeten Sterbewahrscheinlichkeiten. Bei sofort beginnenden Enhanced Annuities findet die Gesundheitsprüfung bei Vertragsbeginn statt, bei aufgeschobenen Renten hingegen am Ende der Ansparphase. Versicherte, die nicht zur Gesundheitsprüfung erscheinen, erhalten in diesem Fall die selbe Rente wie die gesündeste Klasse der Versicherten.

Die bestehende Literatur zum Thema Enhanced Annuities ${ }^{5}$ konzentriert sich in erster Linie auf den britischen Markt. In diesem Zusammenhang werden Entwicklung und Größe des Marktes sowie das Marktpotential untersucht. Des Weiteren existieren Beschreibungen verschiedener Arten von Enhanced Annuities, möglicher Underwriting Methoden und Herausforderungen für das Underwriting, einer möglichen Rückversicherung sowie Diskussionen steuerlicher und vertrieblicher Aspekte, etc. Darüber hinaus führt Ainslie (2000) eine theoretische Analyse von Antiselektionseffekten auf Anbieter traditioneller Rentenversicherungsprodukte durch. Er betrachtet ein hypothetisches Portfolio von Männern im Alter 65, dessen Sterblichkeitsheterogenität er mit Hilfe einer Normalverteilung beschreibt. Für verschiedene Parametrisierungen dieser Verteilung bestimmt er den Anteil von Rentnern, die Enhanced Annuities an Stelle herkömmlicher Ren-

3 Vgl. z. B. Ainslie, R. (2000), S. 6, Hamdan, S. / Rinke, C.-R. (2006), S. 5 oder Jones, G. / Richards, S. (2004), S. 20.

4 Den Autoren sind lediglich zwei Produkte am deutschen Markt bekannt: Die Extra Rente der LV 1871 und die DSP-Vorzugsrente.

5 Vgl. z. B. Ainslie, R. (2000), Hamdan, S. / Rinke, C.-R. (1998), Jones, G. / Richards, S. (2004), S. 20 ff., Richards, S. / Robinson, D. (2005), Rinke, C.-R. (2002), Weinert, T. (2006). 
tenprodukte kaufen würden. Daneben betonen z. B. Jones und Richards (2004) das Risiko des Underwritings von Enhanced Annuities ${ }^{6}$, allerdings ohne quantitative Analysen durchzuführen.

Es existieren in der Literatur noch keine Ansätze zur Modellierung des individuellen Underwritings von Enhanced Annuities, der Qualität des Underwritings und zur Analyse der Auswirkungen auf die Risiko- und Gewinnsituation eines Versicherers. Des Weiteren ist der Einfluss von Antiselektion auf die Profitabilität von Anbietern traditioneller Rentenversicherungsprodukte durch das Angebot von Enhanced Annuities anderer Anbieter noch nicht untersucht worden.

Die vorliegende Arbeit führt derartige Analysen durch. Dazu spezifizieren wir die Sterblichkeitsheterogenität in einem Versichertenbestand und die Ergebnisse eines individuellen Underwritings durch geeignete korrelierte Wahrscheinlichkeitsverteilungen. Mit Monte Carlo Methoden wird der Gewinn/Verlust verschiedener Modell-Versicherer simuliert und die Risikosituation eines herkömmlichen Rentenversicherers mit der von Anbietern von Enhanced Annuities mit verschiedener Güte des individuellen Underwritings verglichen. Es erfolgt ebenfalls eine Untersuchung der Auswirkungen von Antiselektion auf einen Standardversicherer.

Die Arbeit gliedert sich wie folgt: In Abschnitt 2. werden als Ausgangsbasis die relevanten Wahrscheinlichkeitsverteilungen modelliert: In Abschnitt 2.1.1 spezifizieren wir die Heterogenität in einem Versichertenbestand durch individuelle Sterbewahrscheinlichkeiten. Hierzu modellieren wir die Wahrscheinlichkeitsverteilung eines so genannten frailty factors, der den Gesundheitszustand jedes Versicherten beschreibt und dessen Sterbewahrscheinlichkeiten determiniert. Abschnitt 2.1.2 widmet sich der Verteilung des frailty factors in einem Versichertenbestand unter dem Einfluss von Antiselektion. In Abschnitt 2.2 modellieren wir das individuelle Underwriting durch eine Verteilung, die mit dem tatsächlichen frailty factor korreliert. Der Korrelationskoeffizient ist dabei ein Maß für die Güte des Underwritings. Abschnitt 2.3 erläutert schließlich, wie in diesem Modellrahmen der Gewinn / Verlust eines Versicherers auf Einzelpolicen- und Bestandsebene bestimmt werden kann. Die Beschreibung von Auswertungen und Ergebnissen erfolgt in Abschnitt 3. Zunächst werden in Abschnitt 3.1 die Parameter festgelegt. Durch Analyse der empirischen Gewinnverteilung vergleichen wir in Abschnitt 3.2.1 traditionelle Versicherer mit Anbietern von Enhanced Annuities. Insbesondere zeigen wir den Einfluss der Qualität des individuellen Underwritings auf die Risikosituation eines Versicherungsunternehmens und untersuchen den Einfluss von Antiselektion in Abschnitt 3.2.2 Wir schließen in Abschnitt 4. mit einer kurzen Zusammenfassung.

6 Vgl. Jones, G. / Richards, S. (2004), S. 20. 


\section{Modellierung}

Um die Notation zu vereinfachen, konzentrieren wir uns im Folgenden auf eine sofort beginnende lebenslange Leibrente. Unsere Ergebnisse sind jedoch auf den Fall einer aufgeschobenen Rentenversicherung mit Underwriting am Ende der Ansparphase übertragbar. Wir berücksichtigen keine Betriebskosten. In den folgenden Abschnitten wird eine vereinfachte Übersicht über unser Modell gegeben. Für weitere Details zur mathematischen Modellierung der Sterblichkeit und ein vollständiges Formelwerk verweisen wir auf Hoermann und Ruß (2007).

\subsection{Die Bestimmung der Leistungsdauer}

\subsubsection{Traditionelle Rentenversicherungen und Enhanced Annuities}

Die Leistungen des Versicherers werden von der individuellen Sterblichkeit des Versicherten bestimmt. Zur Spezifikation der Heterogenität im Versichertenbestand nehmen wir an, dass sich die tatsächliche Sterblichkeit jedes Individuums lediglich durch einen multiplikativen Faktor ${ }^{7}$ (auch als frailty factor, Sterblichkeits- oder Mortalitätsmultiplikator bezeichnet) von einer gegebenen Standardsterbetafel unterscheidet. ${ }^{8}$ Damit ergibt sich die einjährige individuelle Sterbewahrscheinlichkeit als Produkt aus dem Sterblichkeitsmultiplikator $d$ der versicherten Person und den Sterbewahrscheinlichkeiten gemäß Standardsterbetafel. Resultiert ein Wert größer als Eins, so setzen wir die Sterbewahrscheinlichkeit auf Eins. Bei einem Multiplikator kleiner Eins wird die am Ende der Tafel noch verbleibende Wahrscheinlichkeitsmasse dem rechnerischen Höchstalter der Sterbetafel zugeordnet.

Der Parameter $d$ beschreibt somit den Gesundheitszustand eines Individuums. Für $0<d<1$ besitzt die betrachtete Person eine überdurchschnittliche Lebenserwartung. Für $d=1$ stimmt die tatsächliche individuelle Sterblichkeit mit der Sterblichkeit gemäß Sterbetafel überein. Bei einem Faktor $d>1$ ist der Gesundheitszustand beeinträchtigt.

7 Ein derartiger Faktor wird häufig analog auf die stetige Sterblichkeitsintensität angewandt, vgl. Vaupel, J. / Manton, K. / Stallard, E. (1979), S. 440; vgl. auch Jones, B. (1998), S. 81.

8 Vgl. z. B. Pitacco, E. (2003), S. 13. Diese Form der Modellierung ist sinnvoll, da viele Krankheiten, wie beispielsweise Herzkreislauferkrankungen, welche gemäß WHO Europe (2004), S. 12 oder PAN American Health Organization (2006), S. 7 die Haupttodesursache darstellen, die Sterblichkeit generell über einen längeren Zeitraum erhöhen. In der praktischen Anwendung ist $u$. U. die Verwendung unterschiedlicher „Formen“ von Übersterblichkeit für verschiedene Krankheiten angebracht, zum Beispiel eine additive Übersterblichkeit für eine gewisse Anzahl von Jahren. 
Wählt man zufällig ein Individuum aus einer Grundgesamtheit aus, so modellieren wir das zugehörige $d$ als Realisierung einer Zufallsvariablen $D{ }^{9}$ Vereinfacht ausgedrückt beschreibt die Verteilung von $D$, welcher Teil der Grundgesamtheit sich in welchem Gesundheitszustand befindet. Für diese Verteilung (vgl. Abbildung 1) treffen wir die folgenden Annahmen, die auch in der Literatur zu finden sind: ${ }^{10}$

i. Sie ist stetig, so dass feinste Abstufungen des Gesundheitszustandes und damit der verbleibenden Lebenserwartung möglich sind.

ii. Ihr Wertebereich ist positiv.

iii. Ihre Dichte verläuft flach bei Null und durch den Ursprung, weil Sterbewahrscheinlichkeiten nahe bei Null unrealistisch sind.

iv. Die Verteilung ist rechtsschief, d. h. es können sehr große Werte von $d$ vorkommen, nach unten sind diese jedoch begrenzt.

v. Über die Grundgesamtheit ist der Erwartungswert gleich Eins, d. h. im Mittel verhält sich die Sterblichkeit gemäß Sterbetafel.

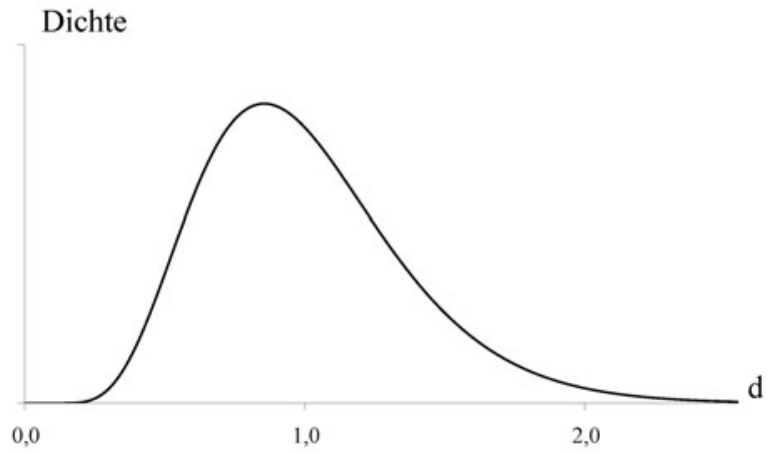

Abbildung 1: Skizze einer möglichen Verteilung der Unter- und Übersterblichkeit in der Bevölkerung, die die gewünschten Eigenschaften besitzt

\subsubsection{Traditionelle Rentenversicherungen bei Antiselektion}

Falls es in einem Markt Anbieter von Enhanced Annuities gibt, so ist davon auszugehen, dass lediglich schlechte Risiken bei einem Versicherer, der

9 Vgl. Czernicki, D. / Harewood, N. / That, M. (2003). Im stetigen Kontext geht die Modellierung von z. B. Jones, B. (1998), S. $80-83$ oder Pitacco, E. (2003), S. 14 zurück auf Vaupel, J. / Manton, K. / Stallard, E. (1979), S. 440.

10 Vgl. Pitacco, E. (2003), S. 14, Butt, Z. / Haberman, S. (2002), S. 5, Hougaard, P. (1984), S. 75 und 79, Ainslie, R. (2000), S. 44, Czernicki, D. / Harewood, N. / That, M. (2003), S. 5. 
ausschließlich Standardrenten vertreibt, abschließen. ${ }^{11}$ In der Realität wird sich jedoch aus verschiedenen Gründen nur ein Teil der Kunden mit reduzierter Lebenserwartung tatsächlich für Enhanced Annuities entscheiden. Dies wird in unserem Modell dadurch berücksichtigt, dass wir davon ausgehen, dass lediglich ein gewisser Anteil in Höhe von $s \%$ aller Personen mit einem Sterblichkeitsmultiplikator $d$ über einer definierten Schranke $d^{*}$ eine Enhanced Annuity einer Standardrente vorzieht. Die resultierende modifizierte Sterblichkeit im Portfolio eines traditionellen Anbieters wird repräsentiert durch die Zufallsvariable $\tilde{D}$.

\subsection{Die Bestimmung der Leistungshöhe}

\subsubsection{Traditionelle Rentenversicherungen}

Ein Anbieter traditioneller Rentenversicherungsprodukte nimmt kein individuelles Underwriting (vgl. 2.2.2) vor und kalkuliert die Rente auf Basis der Standardsterbetafel. Somit stimmt die Rentenhöhe bei gleichem Alter, Geschlecht und Einmalbeitrag für alle versicherten Personen überein.

\subsubsection{Enhanced Annuities}

Die Rentenhöhe von Enhanced Annuities wird mit individuellen kalkulatorischen Sterbewahrscheinlichkeiten berechnet. Dazu ermittelt das individuelle Underwriting eine Schätzung $\hat{d}$ für den Mortalitätsmultiplikator $d^{12}$, mit dessen Hilfe sich die kalkulatorischen Sterbewahrscheinlichkeiten analog zur Berechnung der tatsächlichen individuellen Sterbewahrscheinlichkeiten herleiten lassen.

Der Parameter $\hat{d}$ wird modelliert als Realisierung einer Zufallsvariable $\hat{D}$, und wir nehmen an, dass $D$ und $\hat{D}$ die selbe Verteilung besitzen. Somit gibt es keinen systematischen Underwritingfehler, d. h. die Sterblichkeitsschätzung des Underwritings ist über die Grundgesamtheit sowohl im Mittel als auch in Bezug auf den Anteil von Personen, die in einem bestimmten „Krankheitsbereich“ liegen, korrekt. Um uns darüber hinaus ausschließlich auf den Effekt des individuellen Underwritings konzentrieren zu können, berücksichtigen wir keine Sicherheitszuschläge. ${ }^{13}$ Schließlich seien die $\mathrm{Zu}$ fallsvariablen $D$ und $\hat{D}$ positiv korreliert, wobei der Korrelationskoeffizient als Maß für die Güte des Underwritings zu interpretieren ist. Je größer die

11 Vgl. z. B. Zweifel, P. / Eisen, R. (2003), S. 295. S. 13 .

12 Dies ist ein so genanntes „numerical rating system“, vgl. Pitacco, E. (2003),

13 Es könnten auch Sicherheitszuschläge in das Modell integriert werden, indem man kalkulatorische Sterbewahrscheinlichkeiten verwendet, die die aus dem Underwriting resultierenden Sterblichkeiten übersteigen. 
Korrelation, desto kleiner ist die mittlere Abweichung zwischen $d$ und $\hat{d}$. Ein Korrelationskoeffizient von Eins impliziert $d=\hat{d}$; dies entspricht dem hypothetischen Fall eines ,perfekten“ Underwritings.

Es ist zu beachten, dass unser Modell einen stetigen Wertebereich für $\hat{d}$ vorsieht, und dass es in dem Sinne symmetrisch ist, als Versicherte mit erhöhter oder reduzierter Lebenserwartung analog behandelt werden. Bei der praktischen Anwendung wäre u. U. ein diskretes Modell vorzuziehen, welches beispielsweise nur ganzzahlige Vielfache von 0,25 für $\hat{d}$ vorsieht. Alternativ oder zusätzlich könnte man ein asymmetrisches Modell wählen, in welchem alle Versicherten, die eine überdurchschnittliche Lebenserwartung besitzen, in einer Klasse zusammengefasst werden, und lediglich für gesundheitlich beeinträchtigte Personen ein individuelles Underwriting durchgeführt wird.

\subsection{Gewinn pro Police und im Portfolio}

Tabelle 1 zeigt, von welcher Wahrscheinlichkeitsverteilung Leistungsdauer und (kalkulierte) Leistungshöhe für verschiedene Modellversicherer abhängen. Hieraus lässt sich der auf den Vertragsbeginn diskontierte, einzelvertragliche Gewinn als Differenz aus der Einmalprämie und den diskontierten zukünftigen Leistungen bestimmen.

Tabelle 1

Vergleich von Leistungsdauer und Leistungshöhe

\begin{tabular}{|l|c|c|}
\hline \multicolumn{1}{|c|}{ Bestand } & $\begin{array}{c}\text { Leistungsdauer } \\
\text { abhängig von }\end{array}$ & $\begin{array}{c}\text { Leistungshöhe } \\
\text { abhängig von }\end{array}$ \\
\hline Traditionelle Rentenversicherungen & $D$ & 1 \\
\hline Enhanced Annuities & $D$ & $\hat{D}$ \\
\hline $\begin{array}{l}\text { Traditionelle Rentenversicherungen } \\
\text { unter dem Einfluss von Antiselektion }\end{array}$ & $\tilde{D}$ & 1 \\
\hline
\end{tabular}

Wir analysieren ein Kollektiv bestehend aus $n$ Versicherten, die alle dasselbe Alter und Geschlecht besitzen. Diese Personen werden zufällig aus einer Grundgesamtheit ausgewählt. Wir gehen davon aus, dass eine Standardsterbetafel verwendet wird, die ein Durchschnittsindividuum dieser Grundgesamtheit beschreibt. Die Versicherten unterscheiden sich durch den Multiplikator, welcher die individuellen Sterbewahrscheinlichkeiten bestimmt. Für den Fall von Enhanced Annuities unterscheiden sie sich auBerdem durch das Ergebnis des Underwritings, also die kalkulatorische Sterbewahrscheinlichkeit, die die Rentenhöhe festlegt. Die beiden Zufalls- 
variablen seien positiv korreliert, wobei der Korrelationskoeffizient für alle Personen gleich ist. Der Gewinn des Versicherers in einem Portfolio ergibt sich dann als Summe der Gewinne der Einzelpolicen. Im folgenden Abschnitt 3. wird die Verteilung zukünftiger Gewinne für die verschiedenen Versichertenbestände mit Hilfe einer Monte Carlo Simulation approximiert und analysiert.

\section{Auswertungen}

\subsection{Wahl der Parameter und Simulation}

Sofern nicht anders angegeben, konzentrieren wir uns im Folgenden auf ein Versichertenportfolio bestehend aus 100 männlichen versicherten Personen im Alter 65. Die Einmalprämie beträgt $100.000 €$ pro versicherte Person, und wir verwenden den derzeitigen Garantiezins für deutsche Rentenversicherungsprodukte in Höhe von $2,25 \%$. Wir gehen davon aus, dass der Versicherer auch immer genau diesen Zins erwirtschaftet, d. h. der Gewinn oder Verlust resultiert lediglich aus einer Abweichung der tatsächlichen Anzahl Todesfälle von der kalkulierten. Eine solche Abweichung resultiert einerseits aus Zufallsschwankungen andererseits aus Unterschieden zwischen den individuellen und den kalkulatorischen Sterbewahrscheinlichkeiten. Für Enhanced Annuities werden die Ergebnisse bei unterschiedlicher Qualität des individuellen Underwritings verglichen, d. h. wir betrachten verschiedene Werte des Korrelationskoeffizienten. Bei der Berücksichtigung von Antiselektion werden ebenfalls unterschiedliche Selektionsintensitäten gewählt. Als Standardsterbetafel für unsere Analysen verwenden wir die DAV2004R Sterbetafel ${ }^{14}$.

Zur Verteilung der Unter- und Übersterblichkeit in der Bevölkerung gibt es unseres Wissens - insbesondere für Senioren - keine Daten. Eine Ableitung einer angemessenen Verteilung aus epidemiologischen oder medizinischen Daten erscheint daher derzeit nicht möglich. Deshalb leiten wir eine geeignete Verteilung aus den in Abschnitt 2.1.1 aufgeführten Eigenschaften ab. Eine Verteilung, die diese Eigenschaften besitzt und die in der Literatur häufig zur Beschreibung der Verteilung des Sterblichkeitsmultiplikators in der Bevölkerung verwendet wird, ist die dreiparametrige Gammaverteilung: ${ }^{15} \Gamma(\alpha, \beta, \gamma)$. Da bestimmte Unfälle unvermeidlich und daher Sterbewahrscheinlichkeiten nahe bei Null unrealistisch sind, wählen wir ei-

14 Die DAV2004R Sterbetafel ist die aktuellste deutsche Rententafel für die Alter 0 bis 121. Wir verwenden die Tafel mit Altersverschiebung für die Geburtsjahrgänge 1910 bis 2020. Für weiterführende Informationen vgl. DAV (2005).

15 Vgl. Jones, B. (1998), S. 82, Pitacco, E. (2003), S. 16, Butt, Z./Haberman, S. (2002), S. 8-9, Hougaard, P. (1984), S. 76; alle basierend auf Vaupel, J. / Manton, K. / Stallard, E. (1979), S. $441-442$. Details zur Gammaverteilung, vgl. Casella, G. / Berger, R. (2002), S. $99-100$. 
nen positiven dritten Parameter $\gamma \cdot{ }^{16}$ Unter Berücksichtigung der Eigenschaften aus Abschnitt 2.1.1 ist beispielsweise $\alpha=3, \beta=0,2$ und $\gamma=0,4$ eine plausible Parameterkombination. Wir betrachteten ferner eine Reihe unterschiedlicher Parametrisierungen, die eine Verteilung mit den in Abschnitt 2.1.1 geforderten Eigenschaften erzeugen. Alle diese Verteilungen führten zu ähnlichen Ergebnissen.

Zur Approximation der Wahrscheinlichkeitsverteilung zukünftiger Gewinne in einem Portfolio führen wir 10.000 Monte Carlo Simulationen durch. In jedem Simulationsdurchgang werden die Rentenhöhe, die Dauer der Rentenzahlung und damit der Gewinn des Versicherers pro Police und kumuliert über alle Versicherten berechnet. Dazu müssen für jede versicherte Person die folgenden Zufallszahlen erzeugt werden: $d$ bzw. $\tilde{d}$ spezifiziert die individuelle Sterblichkeit und damit die Wahrscheinlichkeitsverteilung der Leistungsdauer (d. h. die verbleibende Lebensdauer eines Versicherten). Das individuelle Underwriting bestimmt den Sterblichkeitsmultiplikator $\hat{d}$, welcher die kalkulatorischen Sterbewahrscheinlichkeiten und damit die Rentenhöhe einer Enhanced Annuity festlegt. Eine Realisierung der individuellen Sterbewahrscheinlichkeit dient schließlich zur Bestimmung des Todeszeitpunkts innerhalb jedes Simulationspfades.

\subsection{Ergebnisse}

Zum Vergleich der Gewinnsituation und Risikostruktur eines traditionellen Rentenversicherers und eines Anbieters von Enhanced Annuities analysieren wir die empirische Verteilung des Gewinns bzw. Verlusts und betrachten insbesondere den Erwartungswert, die Standardabweichung und Quantile der Verteilung. Dabei konzentrieren wir uns auf den Einfluss der Qualität des Underwritings. Im Anschluss werden die Auswirkungen von Selektionseffekten auf einen Standardversicherer durch das Konkurrenzangebot von Enhanced Annuities untersucht. ${ }^{17}$

\subsubsection{Vergleich traditioneller Rentenversicherungen mit Enhanced Annuities}

Wir kalibrieren unser Modell so, dass der erwartete Gewinn eines Anbieters traditioneller Rentenversicherungen Null beträgt. Abbildung 2 illustriert den erwarteten Gewinn eines Bestands von Enhanced Annuities in Abhängigkeit der Qualität des Underwritings und vergleicht ihn mit dem

16 Vgl. Hougaard, P. (1984), S. 79.

17 Die aufgeführten Ergebnisse unterscheiden sich von den Resultaten in Hoermann, G./Ruß, J. (2007) u. a. dadurch, dass eine alternative Parametrisierung der Verteilung der Unter- und Übersterblichkeit gewählt wurde. 
entsprechenden Wert eines Standardversicherers. Dieser Vergleich zeigt, dass bereits bei eher geringer Güte des individuellen Underwritings, d. h. bei einem Korrelationskoeffizienten von Null, der erwartete Gewinn aus Enhanced Annuities den erwarteten Gewinn aus traditionellen Produkten deutlich übersteigt. Der Effekt beruht lediglich auf der Berücksichtigung der Sterblichkeitsverteilung im Portfolio bei der Kalkulation von Enhanced Annuities. Mit anderen Worten ist das Angebot von Enhanced Annuities mit individuellem Underwriting sogar unter der schwachen Annahme, dass die Einschätzung lediglich im Durchschnitt korrekt ist, vorteilhaft für den erwarteten Gewinn eines Versicherers. Der positive Effekt und damit der erwartete Gewinn steigt mit der Qualität des Underwritings: er verdoppelt sich nahezu von etwa 0,8\% des Prämienvolumens bei einer Korrelation von Null auf 1,6\% bei einer Korrelation von Eins.

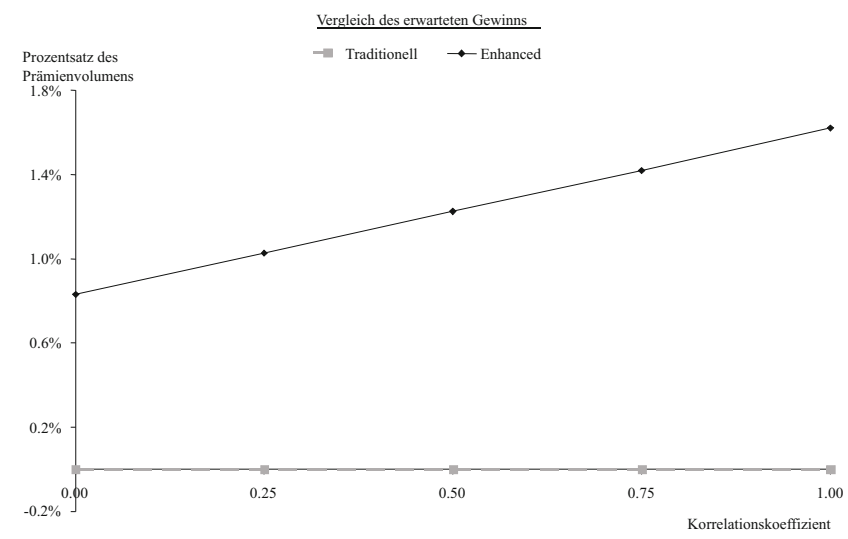

Abbildung 2: Vergleich des erwarteten Gewinns in Prozent des Prämienvolumens in Abhängigkeit des Korrelationskoeffizienten

Im nächsten Schritt wird untersucht, ob die Zunahme des erwarteten Gewinns mit einer Zunahme des Risikos einhergeht. Es zeigt sich, dass dies bei einer geringen Korrelation tatsächlich der Fall ist (vgl. Abbildung 3): die Standardabweichung des Gewinns beginnt bei etwa 2,40\% des Prämienvolumens bei einer Korrelation von Null und übersteigt den Wert des herkömmlichen Rentenversicherers (2,33\%; graue gestrichelte Linie). Die Streuung verringert sich jedoch bei steigendem Korrelationskoeffizienten und nähert sich einem Wert von etwa 2,20\% bei einer Korrelation von Eins an. Bei einer Korrelation von 0,4 und größer hat ein Anbieter von Enhanced Annuities sowohl einen höheren erwarteten Gewinn als auch eine geringere Volatilität als ein Anbieter von Standardrenten. 


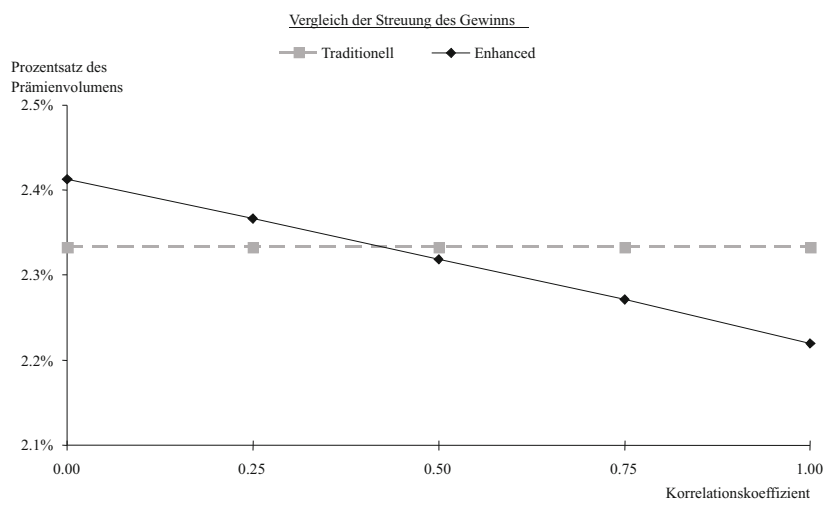

Abbildung 3: Vergleich der Standardabweichung des Gewinns in Abhängigkeit des Korrelationskoeffizienten

Abbildung 4 zeigt die gesamte empirische Verteilung des Gewinns eines Versicherers. Bei Enhanced Annuities ist die Verteilung nach rechts verschoben (d. h. der erwartete Gewinn ist höher) und enger (d. h. die Volatilität ist geringer) als bei herkömmlichen Rentenprodukten.

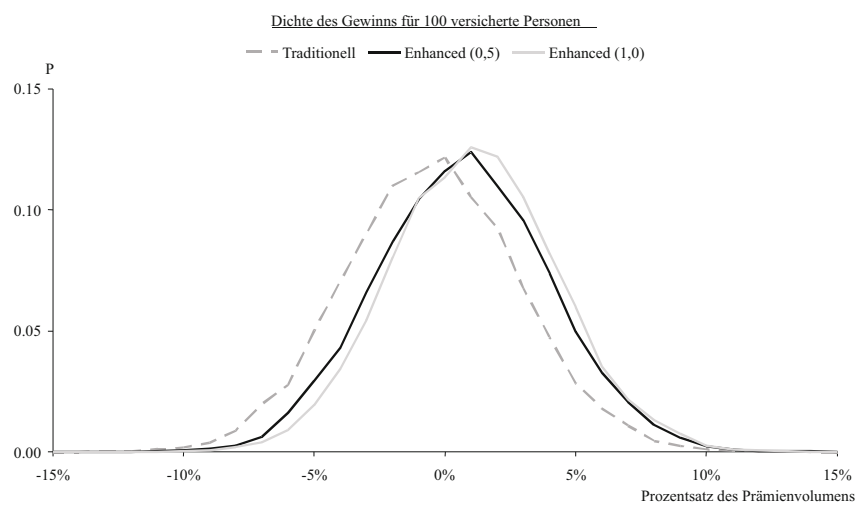

Abbildung 4: Vergleich der Dichte des Gewinns für 100 versicherte Personen

Die Wahrscheinlichkeit für einen positiven Gewinn ${ }^{18}$ des traditionellen Versicherers liegt bei $50 \%$; bereits bei einem Korrelationskoeffizienten von Null ist sie für den Anbieter von Enhanced Annuities auf $60 \%$ erhöht, bei

18 Unter Berücksichtigung von Sicherheitszuschlägen jeglicher Art wären diese Werte für beide Anbieter höher. 
einer Korrelation von 0,5 beträgt sie $64 \%$ und bei einer Korrelation von Eins sogar $69 \%$. Mit einer Wahrscheinlichkeit von $5 \%$ ist der Verlust des Standardversicherers größer als $5,4 \%$ des Prämienvolumens. Für das Enhanced Annuity Portfolio mit einer Korrelation von 0,5 reduziert sich das $5 \%$-Quantil auf 4,2\%; bei einer Korrelation von Eins beträgt es nur noch $3,5 \%$ der gesamten Prämieneinnahmen.

Eine Variation der Bestandsgröße liefert die erwarteten Ergebnisse: Der Vergleich von Abbildung 5 (Ergebnisse für 400 Versicherte) mit Abbildung 4 (100 Versicherte) zeigt, dass für beide Versicherer der Bereich von Zufallsschwankungen bei einer Vervierfachung der Portfoliogröße grob halbiert wird.

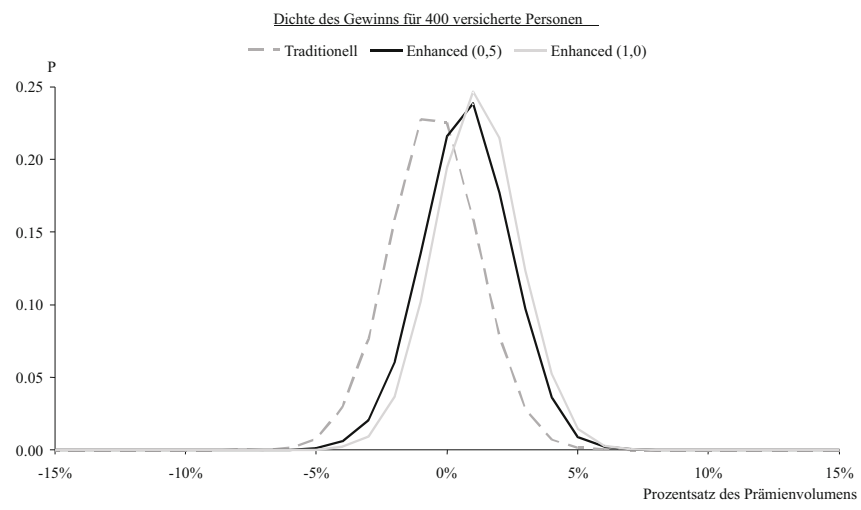

Abbildung 5: Vergleich der Dichte des Gewinns für 400 versicherte Personen

Die Wahrscheinlichkeit für einen positiven Gewinn für den Anbieter von Enhanced Annuities steigt bei dieser Vergrößerung des Portfolios von $60 \%$ auf $69 \%$ bei einer Korrelation von Null, von $64 \%$ auf $78 \%$ bei einer Korrelation von 0,5 und von $69 \%$ auf $85 \%$ bei einer Korrelation von Eins. Für den traditionellen Versicherer wird das oben beschriebene 5\%-Quantil annähernd halbiert (2,8\%), für Enhanced Annuities (Korrelation 0,5) ist es von $4,2 \%$ auf $1,5 \%$ reduziert, und bei einer Korrelation von Eins sinkt der Wert sogar bis auf 1,0\% des Prämienvolumens (verglichen mit 3,5\% bei 100 Versicherten).

\subsubsection{Auswirkungen von Antiselektion}

Abbildung 6 zeigt Ergebnisse unter Berücksichtigung von Antiselektion. Wir nehmen an, dass sich ein gewisser Prozentsatz $s$ aller Personen, deren 
Sterblichkeitsmultiplikator eine „Selektionsschranke“ $d^{*}$ übersteigt, vom traditionellen Versicherer abwendet.

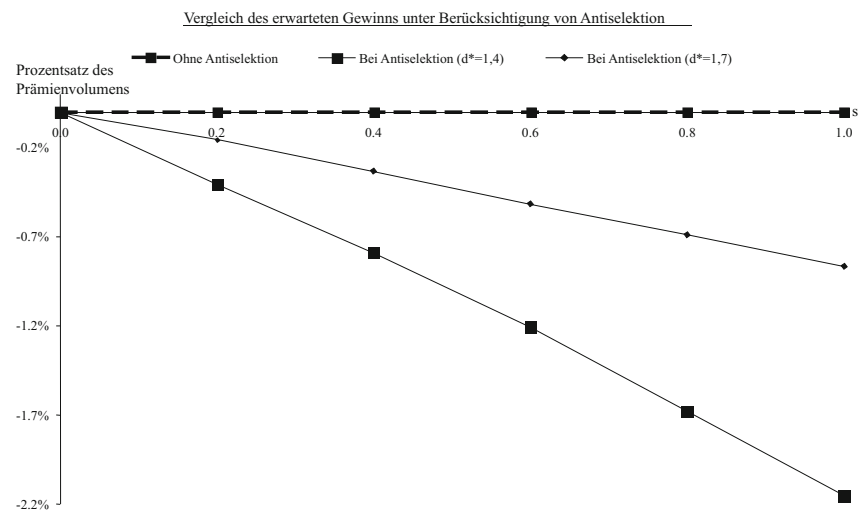

Abbildung 6: Vergleich des erwarteten Gewinns in Abhängigkeit der Selektionsintensität $s$

Abbildung 6 zeigt für verschiedene Werte der Selektionsschranke $d^{*}$, wie sich der erwartete Gewinn des traditionellen Versicherers in Abhängigkeit der Selektionsintensität reduziert. Je höher die Selektionsschranke $d^{*}$, desto weniger Versicherungsanwärter sind von der Selektion betroffen und desto geringer fällt daher die Abnahme des erwarteten Gewinns aus: Für $d^{*}=1,7$ steigt der erwartete Verlust lediglich bis auf maximal $0,9 \%$, für $d^{*}=1,4$ hingegen bis auf $2,2 \%$ des Prämienvolumens.

Diese Analysen zeigen, dass sich für Anbieter herkömmlicher Rentenversicherungsprodukte signifikante Nachteile ergeben können, wenn Wettbewerber Enhanced Annuities anbieten, selbst wenn nur ein Teil der potentiellen Versicherten dieses Angebot wahrnimmt.

\section{Zusammenfassung}

Der vorliegende Beitrag vergleicht das Risikoprofil eines traditionellen Rentenversicherers mit dem eines Anbieters von Enhanced Annuities. Dazu betrachteten wir jeweils einen hinsichtlich individueller Sterbewahrscheinlichkeiten heterogenen Versichertenbestand. Für Enhanced Annuities wurde angenommen, dass das Resultat des individuellen Underwritings mit der individuellen Sterblichkeit korreliert ist. Der Korrelationskoeffizient diente als Maß für die Güte des Underwritings. Mit Monte Carlo Simulationstechniken bestimmten wir die empirische Verteilung zukünftiger Gewinne bzw. Verluste und verglichen einen Bestand traditioneller Rentenver- 
sicherungen mit einem Bestand von Enhanced Annuities mit individuellem Underwriting unterschiedlicher Güte. Zusätzlich betrachteten wir den traditionellen Rentenversicherer unter dem Einfluss von Antiselektion verursacht durch Anbieter von Enhanced Annuities.

Unsere Ergebnisse machen deutlich, dass die Einführung von Enhanced Annuities nicht nur für Versicherte, sondern auch für die Anbieter von Vorteil wäre. Enhanced Annuities bieten allen versicherten Personen ein vergleichbares Preis-Leistungs-Verhältnis. Insbesondere ist der Bezug von lebenslangen Rentenleistungen bei diesem Produkt auch für Kunden mit reduzierter Lebenserwartung attraktiv, wodurch der Versicherte zusätzlich von eventuellen steuerlichen Vorteilen von Rentenprodukten profitieren kann. Somit könnte durch Enhanced Annuities die Akzeptanz von Rentenprodukten in der Bevölkerung erhöht werden.

Außerdem zeigten wir, dass ein Anbieter von Enhanced Annuities, sofern er auch nur in der Lage ist, die durchschnittliche Verteilung der Unter- und Übersterblichkeit unter den Versicherungsanwärtern richtig zu beurteilen, eine im Vergleich zu einem Anbieter traditioneller Rentenversicherungsprodukte erhöhte Profitabilität aufweist. Die Differenz zwischen den Anbietern ist dabei umso größer, je besser die Qualität des Underwritings. Darüber hinaus sinkt die Volatilität zukünftiger Gewinne mit zunehmender Güte des Underwritings und mit wachsender Portfoliogröße. Falls Wettbewerber Enhanced Annuities vertreiben, ergibt sich durch Antiselektion ein signifikanter negativer Effekt auf Standardversicherer, selbst wenn nur ein Teil der Personen mit verschlechtertem Gesundheitszustand Enhanced Annuities herkömmlichen Renten vorzieht. Daraus lässt sich schließen, dass ein Anbieter von Enhanced Annuities ein deutlich besseres Risikoprofil als ein Anbieter traditioneller Rentenversicherungen aufweist.

\section{Literaturverzeichnis}

Ainslie, R. (2000): Annuity and Insurance Products for Impaired Lives, Arbeitspapier, The Staple Inn Actuarial Society, London.

Brown, J., Davidoff, T. und Diamond, P. (2005): Annuities and Individual Welfare, in: American Economic Review, 95(5), 1573 - 1590.

Butt, Z. und Haberman, S. (2002): Application of Frailty-Based Mortality Models to Insurance Data, Actuarial Research Paper No. 142, Department of Actuarial Science and Statistics, City University, London.

Casella, G. und Berger, R. (2002): Statistical Inference, Second Edition, Duxbury Press, Pacific Grove, California.

Czernicki, D., Harewood, N. und That, M. (2003): Stochastic Modeling of Mortality, Arbeitspapier präsentiert bei dem Stochastic Modeling Symposium. 
$D A V$ (2005): Herleitung der DAV-Sterbetafel $2004 \mathrm{R}$ für Rentenversicherungen, in: Blätter der Deutschen Gesellschaft für Versicherungs- und Finanzmathematik, $27(2), 199-314$.

Dushi, I. und Webb, A. (2004): Household Annuitization Decisions: Simulations and Empirical Analyses, in: Journal of Pension Economics and Finance, 3(2), 109-143.

Hamdan, S. und Rinke, C.-R. (1998): Enhanced Annuities in the United Kingdom, in: Hannover Re's Perspectives - Current Topics of International Life Insurance, 2.

Hoermann, G. und Ruß, J. (2007): Enhanced Annuities, Individual Underwriting and Adverse Selection - A Solution for the Annuity Puzzle?, Arbeitspapier, Universität St. Gallen.

Hougaard, P. (1984): Life Table Methods for Heterogeneous Populations: Distributions describing the Heterogeneity, in: Biometrika, 171(1), 75-83.

Jones, B. (1998): A Model for Analyzing the Impact of Selective Lapsation on Mortality, in: North American Actuarial Journal, 2(1), 79-86.

PAN American Health Organization (2006): Health Situation in the Americas - Basic Indicators, URL: http: //www.paho.org/English/dd/ais/BI-brochure-2006.pdf [Stand: 17. 03. 2007].

Pitacco, E. (2003): Survival Models in Actuarial Mathematics: From Halley to Longevity Risk, Arbeitspapier, Universität Triest, Triest.

Jones, G. und Richards, S. (2004): Financial Aspects of Longevity Risk, Arbeitspapier, The Staple Inn Actuarial Society, London.

Richards, S. und Robinson, D. (2005): Anti-Selection and Annuity Pricing, in: The Actuary, 5, 32 .

Rinke, C.-R. (2002): The Variability of Life Reflected in Annuity Products, in: Hannover Re's Perspectives - Current Topics of International Life Insurance, 8.

Vaupel, J., Manton, K. und Stallard, E. (1979): The Impact of Heterogeneity in Individual Frailty on the Dynamics of Mortality, in: Demography, 16(3), 439-454.

Weinert, T. (2006): Enhanced Annuities on the Move, in: Hannover Re's Perspectives Current Topics of International Life Insurance, 13.

WHO Europe (2004): Highlights on Health in Germany, URL: http://www.euro. who.int/highlights [Stand: 17. 03. 2007].

Zweifel, P. und Eisen, R. (2003): Versicherungsökonomie, 2., verbesserte Auflage, Springer-Verlag, Berlin, Heidelberg, New York.

\section{Zusammenfassung}

Das Preis-Leistungs-Verhältnis herkömmlicher Rentenversicherungen steigt mit der Lebenserwartung des Versicherten. Derartige Produkte sind damit nur für Personen mit relativ hoher Lebenserwartung attraktiv. Der Nachteil für Versicherte mit reduzierter Lebenserwartung wird durch steuerliche Regelungen, die den Bezug von lebenslangen Renten gegenüber Einmalzahlungen bevorteilen, verstärkt. Mit Hilfe von Enhanced Annuities, bei 
denen die Rente umso höher ist, je geringer die Lebenserwartung der versicherten Person, könnte die Attraktivität von Rentenprodukten erhöht werden. Der vorliegende Beitrag vergleicht das Risikoprofil eines traditionellen Rentenversicherers mit dem eines Anbieters von Enhanced Annuities. Ferner werden die Auswirkungen von Antiselektion auf einen Standardversicherer analysiert. Individuelle Sterbewahrscheinlichkeiten und das Ergebnis des individuellen Underwritings werden mit geeigneten, korrelierten Zufallsvariablen modelliert. Monte Carlo Simulationsmethoden liefern Ergebnisse zum Vergleich der Gewinnsituation eines heterogenen Bestands traditioneller Rentenprodukte und eines Portfolios von Enhanced Annuities bei individuellem Underwriting unterschiedlicher Güte sowie zur Analyse des Einflusses von Selektionseffekten.

\begin{abstract}
The value for money of a standard annuity is the higher, the longer the life expectancy of an insured, and therefore it is only acceptable for persons with an above average life expectancy. The discrepancy is intensified by tax regulations that favor lifelong annuity payments opposed to a lump sum. This discrimination of impaired insureds could be prevented if so-called enhanced annuities were offered, i.e. products where the annuity paid is the larger, the lower the person's life expectancy. The article presents a quantitative comparison of the risk profile of insurance companies offering standard annuity contracts compared to enhanced annuities and an analysis of the impact of adverse selection on a standard insurer. By definition of individual mortality rates a heterogeneous insurance portfolio is specified. Besides we model the individual underwriting of enhanced annuities. A Monte Carlo Simulation provides results to compare the profit/loss situation of a portfolio of traditional annuity products and a portfolio of enhanced annuities with individual underwriting of different quality and to assess the impact of selection effects.
\end{abstract}

